\title{
A NOVEL APPROACH FOR MULTIMEDIA FILES AND DATABASE SYNCHRONIZATION
}

\author{
Khushpreet $\operatorname{Kaur}^{1} \&$ Harpreet Kaur $^{2}$
}

Abstract- A distinctly different concept is that of data synchronization. This refers to the need to keep multiple copies of a set of data coherent with one another. Data synchronization is the process of establishing consistency among data from a source to target data storage and vice versa and the continuous harmonization of the data over time. The objective of the research is to provide an algorithm to solve the problem that when all clients are reliant on a single server. If that database becomes unavailable due to planned server downtime or from server failures, all of the remote workers will be disconnected from their data. Data is stored on to their system (user system). When the user connected to the internet data automatically sink from their client system to the server in serial order.

Keywords - Database, Synchronization, DBMS, Client, Server, Image.

\section{INTRODUCTION}

A database can be summarily depicted as a store for data[1]. This makes clarifies building databases is truly a continuation of a human action that has existed since composing started; it can be connected to the aftereffect of any accounting or recording action that happened some time before the approach of the PC period. Be that as it may, this depiction is excessively obscure for some of our motivations, and we refine it as we come.

The formation of a database is required by the operation of an undertaking. We utilize the term enterprise to designate an assortment of tries that range from an aircraft, a bank, or an assembling organization to a stamp gathering or monitoring individuals to whom you need to compose New Year cards.

A gathering of data sorted out such that a PC program can rapidly choose wanted bits of information. You can think about a database as an electronic recording framework.

In software engineering, synchronization insinuates one of two unmistakable yet related ideas: synchronization of process, and synchronization of data[2]. Process synchronization insinuates the possibility that various procedures are to sign up or handshake at one point, keeping in mind the end goal to achieve an assertion or focus on a specific succession of activity. Data synchronization implies keeping numerous duplicates of a dataset in rationality with each other, or to keep up information trustworthiness. Process synchronization primitives are normally used to actualize data synchronization.

\subsection{The Architecture of Database Systems}

Software systems [3] by and large have a design, ie. having of a structure (shape) and association (work). The previous portrays identifiable parts and how they identify with each other fundamentally; the last depicts how the elements of the different auxiliary segments associate to give the general usefulness of the framework in general. Since a database framework is essentially a product framework (but unpredictable), it too has an engineering. A run of the mill engineering must characterize a specific setup of an association between data, software modules, meta-data, interfaces and languages (see Figure 1).

The architecture of a database system decides its ability, unwavering quality, viability and productivity in meeting client necessities. However, other than the obvious capacities seen through some data manipulation language, good database architecture should provide:

a) Independence of data and programs

b) Ease of system design

c) Ease of programming

d) Powerful query facilities

e) Protection of data

\footnotetext{
${ }^{1}$ Department of Computer Science Engineering, Bahra Group of Institutions, Patiala Campus, Punjab, India

${ }^{2}$ Department of Computer Science Engineering, Bahra Group of Institutions, Patiala Campus, Punjab, India
} 


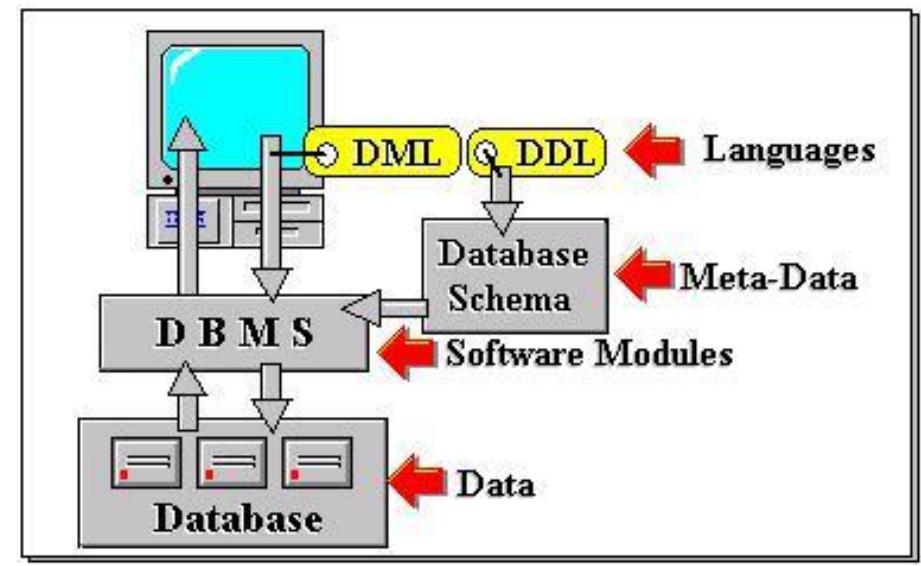

Figure 1. General database system architecture

\section{LITERATURE REVIEW}

\subsection{Fast PDA Synchronization Using Characteristic Polynomial Interpolation (2002)}

In this paper [4] authors(Ari Trachtenberg et al.) had explained that Modern Personal Digital Assistant (PDA) architectures regularly use a discount information exchange convention known as "moderate match up" for synchronizing PDAs with Personal Computers (PCs). This approach is notably wasteful, as far as data transmission utilization and inertness, since the PDA and PC ordinarily share various normal records. We propose, investigate, and actualize a novel PDA synchronization scheme (CPIsync) in view of late data theoretic research. The remarkable property of this plan is that its correspondence many-sided quality depends just on the quantity of contrasts between the PDA and PC, as opposed to the general sizes of their databases. In addition, our execution demonstrates that the computational many-sided quality of CPIsync is handy, and that the general inertness is ordinarily substantially littler than that of moderate match up. In this way, CPIsync has potential for altogether enhancing synchronization conventions for PDAs and, all the more by and large, for heterogeneous frameworks of various machines.

\subsection{Advanced synchronization techniques for complex flow field investigations by means of PIV (2001)}

In this paper [5] authors(B. Stasicki et al.) had explained that Since PIV turned into a develop innovation for stream field examinations in mechanical research, see for example Kompenhans et al. (1998), it has been progressively tested by more confound applications. To the extent static models in wind burrows are concerned, the investigation does not should be synchronized with the measuring instrument. In any case, for the examination of dynamic frameworks synchronization ends up plainly critical. As of late, the persistent however exchanging and, as to the estimation framework, nonconcurrent wake stream of a helicopter's rotor sharp edge was interfaced with a PIV framework so as to research characterized planes in the rotor cutting edges edge of reference, see Raffel et al. (1998). This test is the first of two cases which are portrayed in this paper. Inside the system of the European research program WAVENC (WAke Vortex development and wake vortex ENCounter, Brite/EuRam venture BE97-4112) PIV has been associated with a broken investigation, i.e. the wake stream of a free flying aircraft display in the sling office of ONERA, Lille. The objective was to give a test database to the far-wake advancement of the trailing vortices. The information base will be used to approve CFD estimations. In the first place aftereffects of the PIV estimations and a correlation with smoke perception tests have just been exhibited by Dieterle et al. (1999a). A general depiction of the PIV tests is given by Dieterle et al. (1999b). Next to these two applications a specialized depiction of the synchronization hard-and programming which has been produced in participation DLR in Göttingen and HARDsoft and which is dispersed in permit by PIVTEC will be introduced.

\subsection{Database Replication Techniques: a Three Parameter Classification (2000)}

In this paper [7] authors (Matthias Wiesmann et al.) had explained that data replication is an inexorably critical point as databases are increasingly conveyed over groups of workstations. One of the difficulties in database replication is to present replication without seriously influencing execution. Because of this trouble, current database items use lethargic replication, which is extremely productive however com-guarantee consistency cans. As an option, anxious replication ensures consistency however most existing conventions have a restrictive cost. With a specific end goal to illuminate the flow cutting edge and open up new roads for examine, this paper examinations existing excited procedures utilizing three key parameters. In our investigation, we recognize eight classes of enthusiastic replication conventions and, for every classification, talk about its prerequisites, abilities, and cost. The commitment lies in indicating when anxious replication is plausible and in spelling out the distinctive viewpoints a database replication convention must record for. 


\subsection{An Algorithm to synchronize the local database with cloud Database}

In this paper authors( Saurav kumar Jha et al.) discussed that since the cloud computing [6] stage is generally acknowledged by the business, assortment of uses are planned focusing to a cloud stage. Database as a Service (DaaS) is one of the intense stage of distributed computing. There are many research issues in DaaS stage and one among them is the information synchronization issue. There are many methodologies recommended in the writing to synchronize a neighborhood database by being in cloud condition. Shockingly, not very many work just accessible in the writing to synchronize a cloud database by being in the neighborhood database. The point of this paper is to give a calculation to take care of the issue of information synchronization from nearby database to cloud database.

\section{RESULTS}

\subsection{Login form}

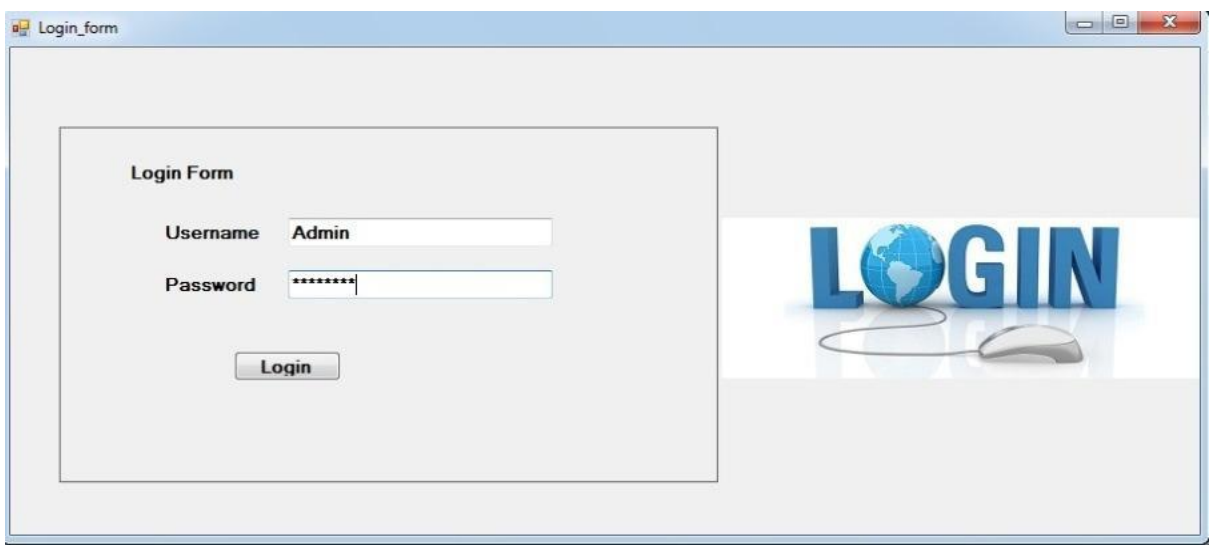

Figure 2. Login form for authentication check

It is a login form for authentication Check. As shown in figure 2 by entering valid username and password user will be rediredted on main page.

\subsection{Main Form}

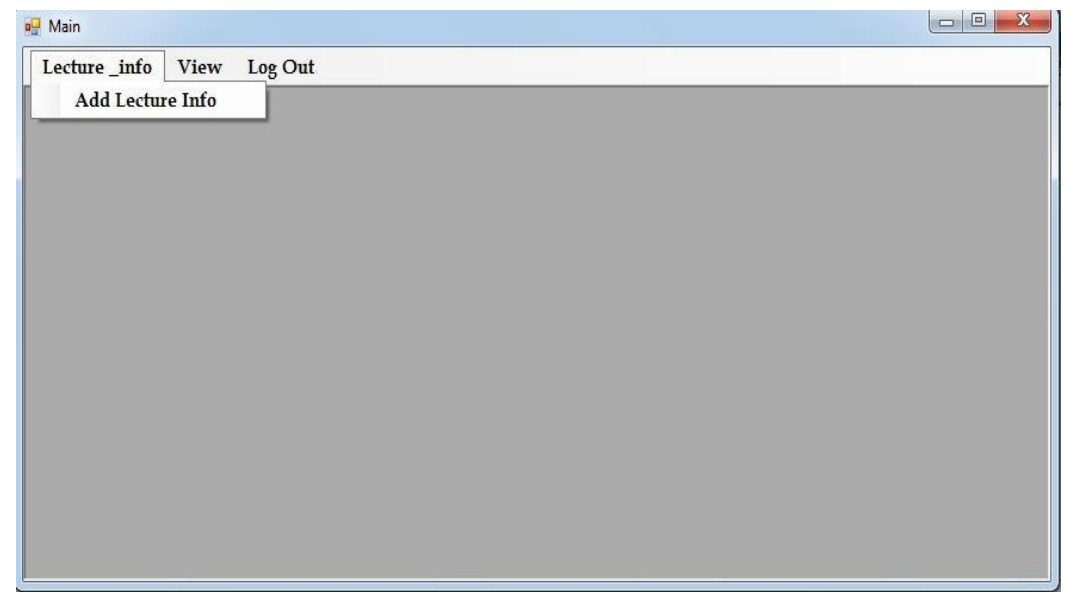

Figure 3. Main Form having all the options of Lecture Details

As shown in figure 3. it is a main form for user to enter lecture information. This form also has option to view entries on client machine as well as on server. 


\subsection{Enter Lecture Information (Offline Mode)}

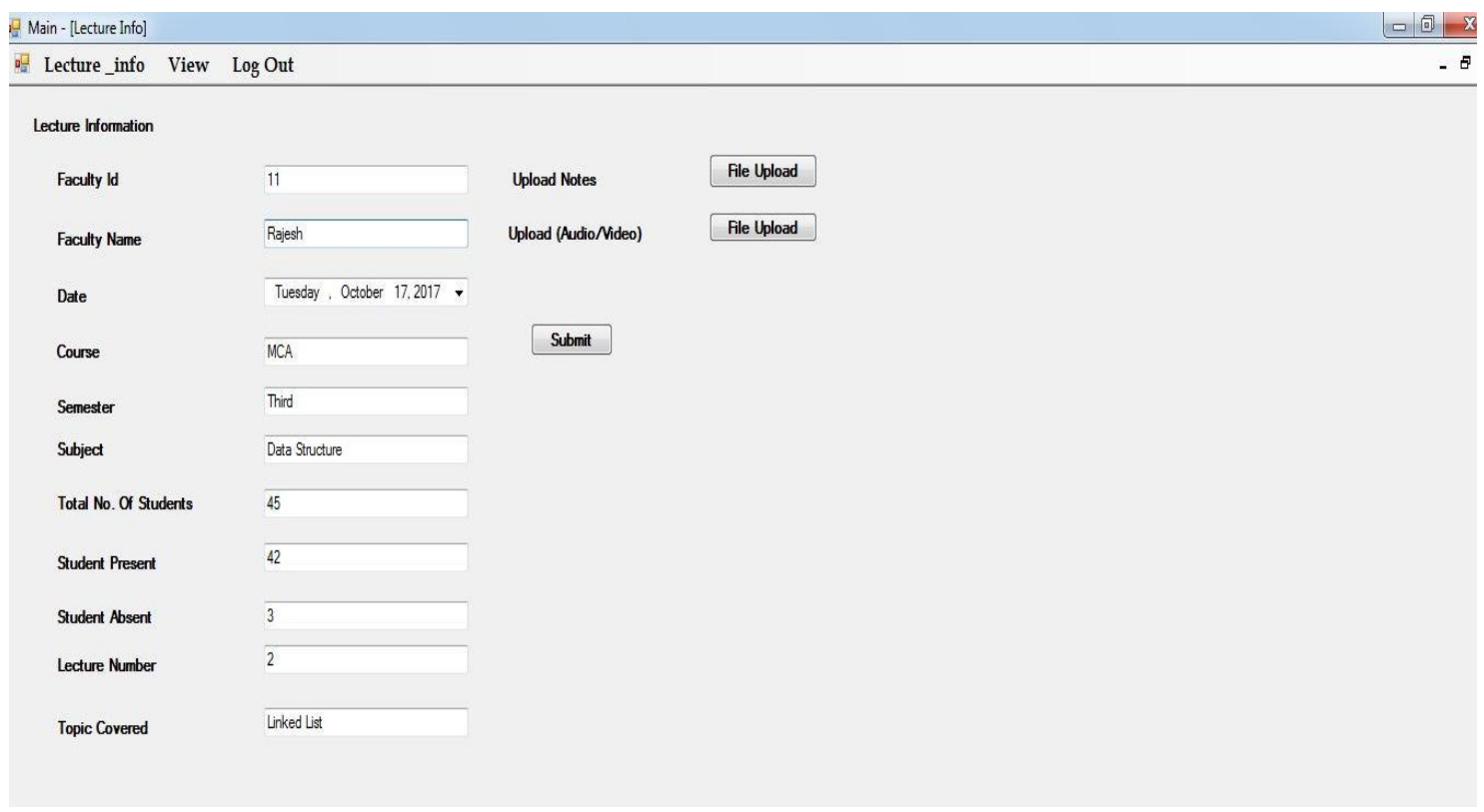

Figure 4 Entering Lecture Information(Offline mode)

As shown in figure 4 by entering the Lecture Detail of Rajesh in offline mode, the information is stored in the client database and files are also stored on client machine.

\subsection{Enter Lecture Information (Offline Mode)}

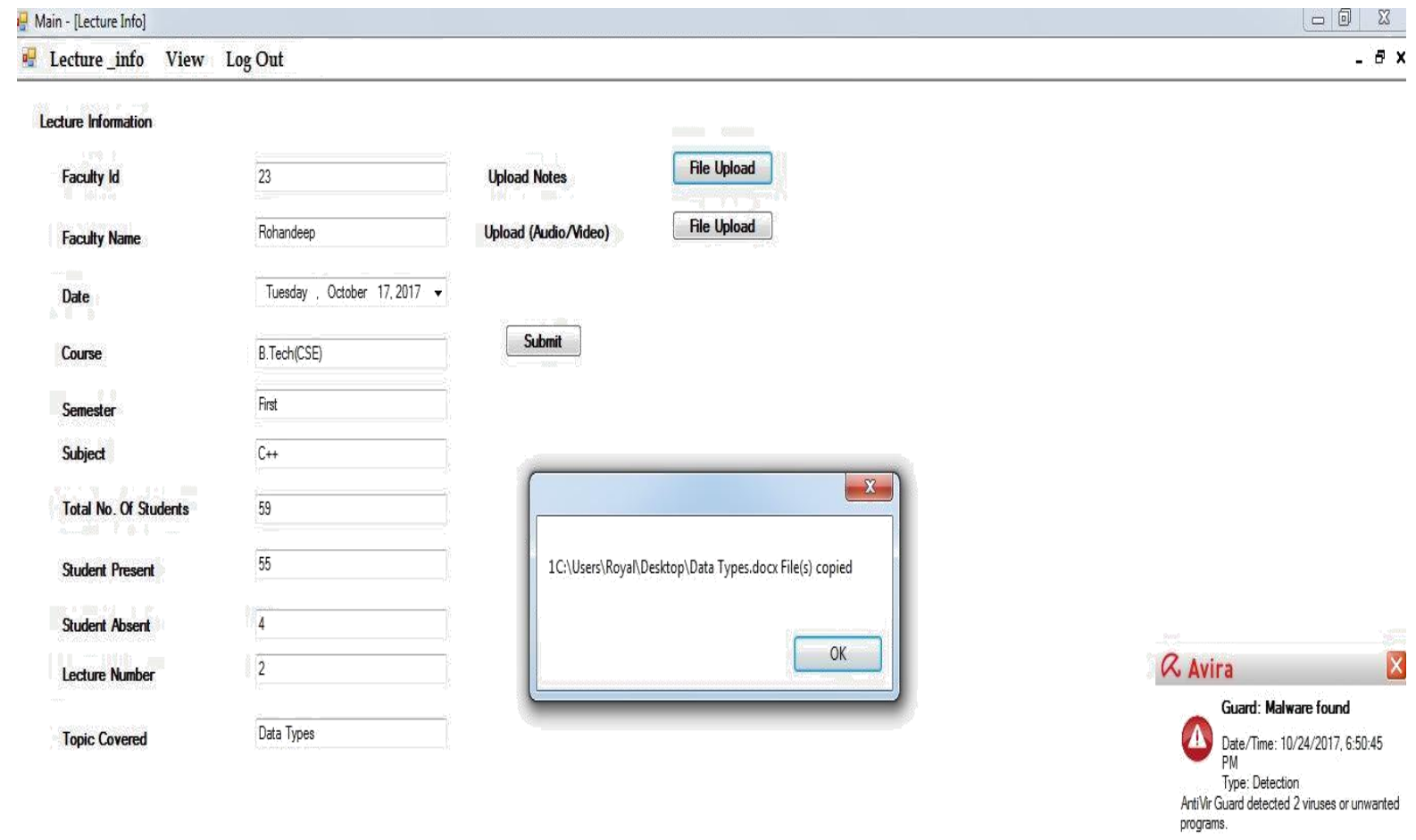

Figure 5. Entering Lecture Information (Offline mode)

As shown in figure 5. by entering the lecture information of Rohandeep in offline mode, Therefore the information is stored in the client database and files are also stored on client system. 
3.5 MS-Access Table (Offline Mode)

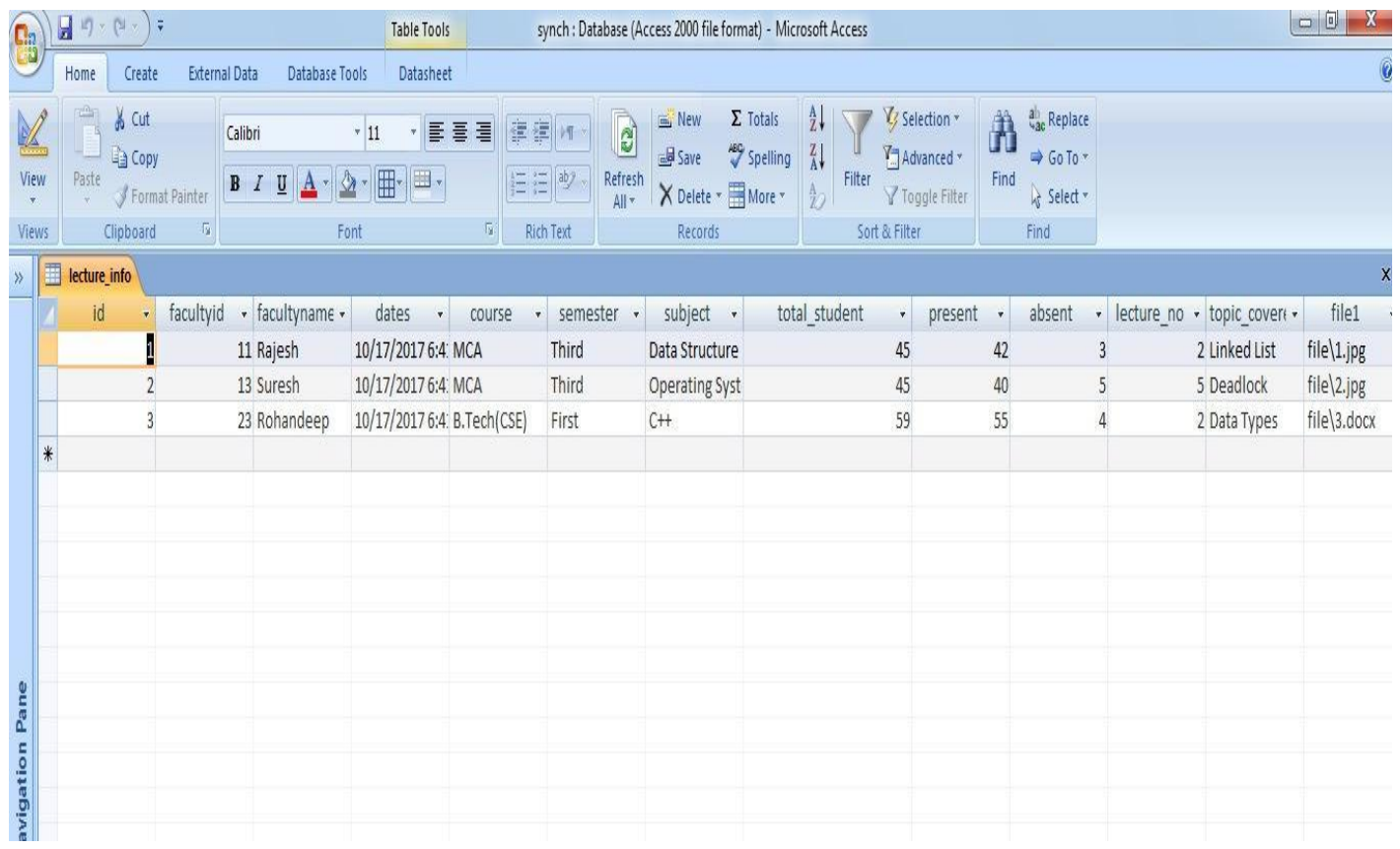

Figure 6. Microsoft access table showing data on client machine

Microsoft access Table Showing data stored on user (Client) system(figure 6). The data entered in the Lecture Information form saved in access database when network is offline.

\subsection{Images in Folder (Offline Mode)}

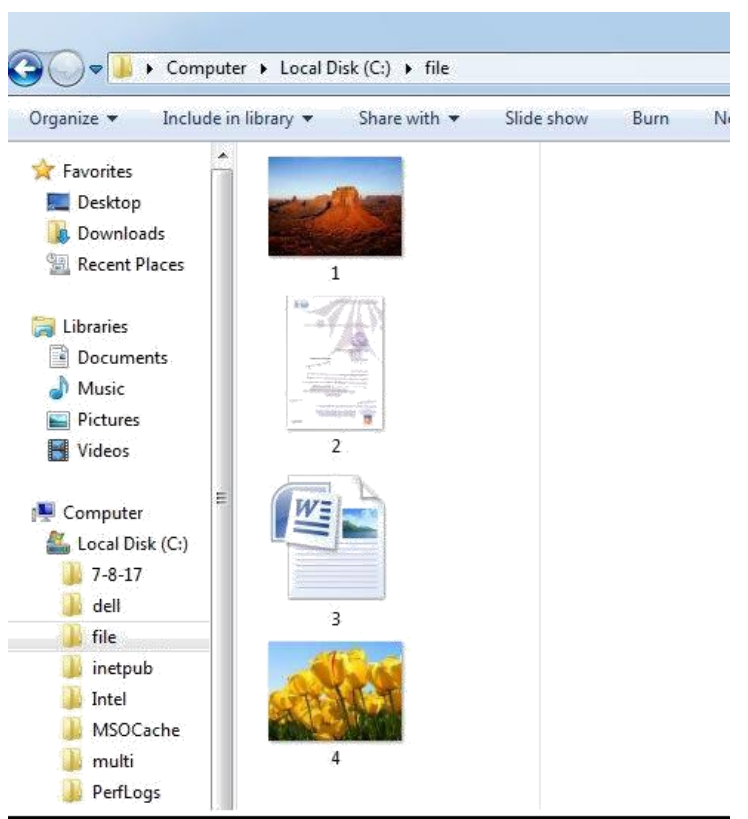

Figure 7. Folder showing uploaded files on client machine

Files are also stored in (user) Client system in a folder(figure 4.6). When files are saved in the folder it renamed from original name to unique name, For example file upload by rohandeep is emp.jpg but after uploading it automatically changed to $3 . j p g$. 


\subsection{Enter Lecture Information (Online Mode)}

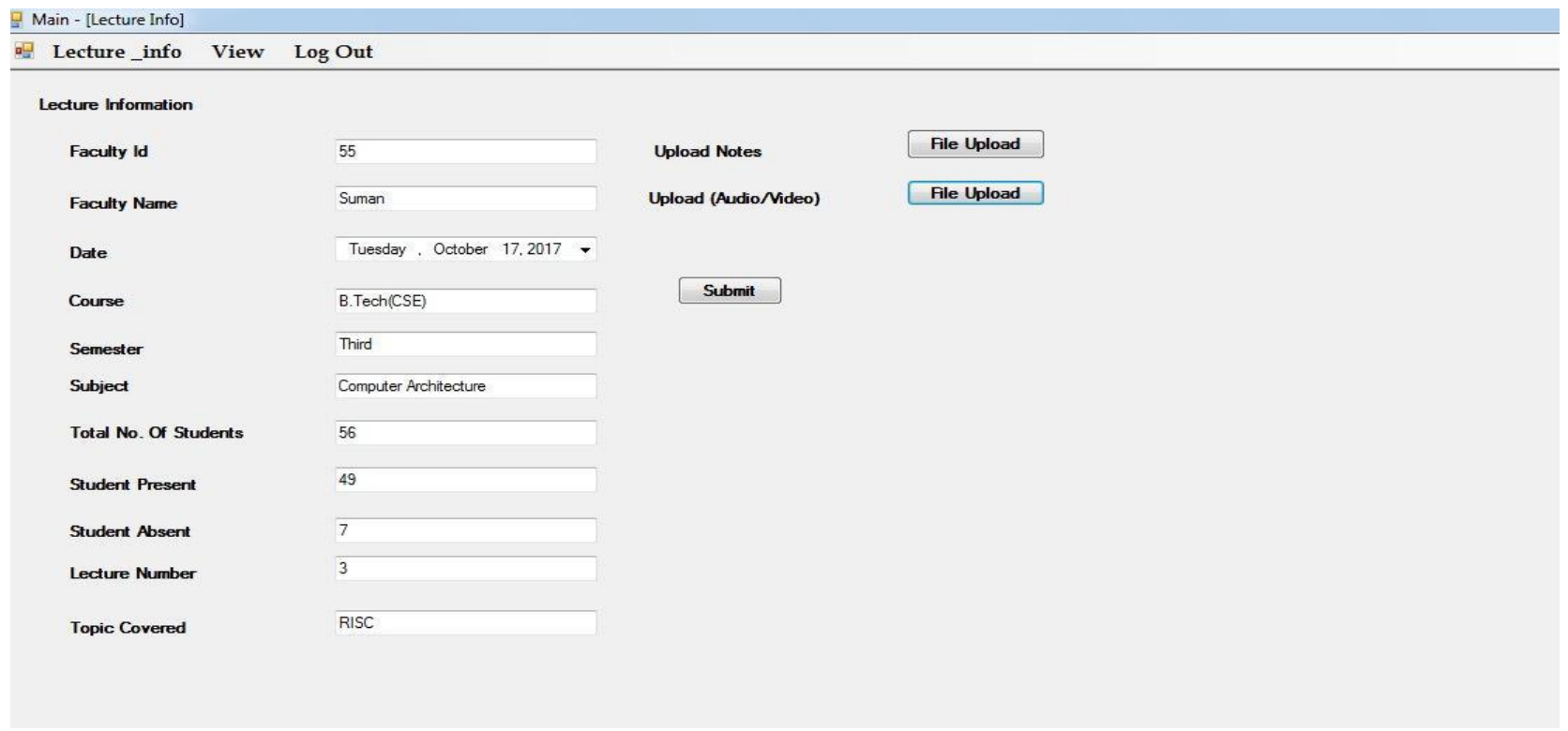

Figure 8. Entering Lecture Information(Online mode)

As shown in figure 8 by entering the lecture information of suman in online mode, therefore the information is stored in the Server database and files are uploaded on server.

\subsection{Data Synchronization (Online Mode)}

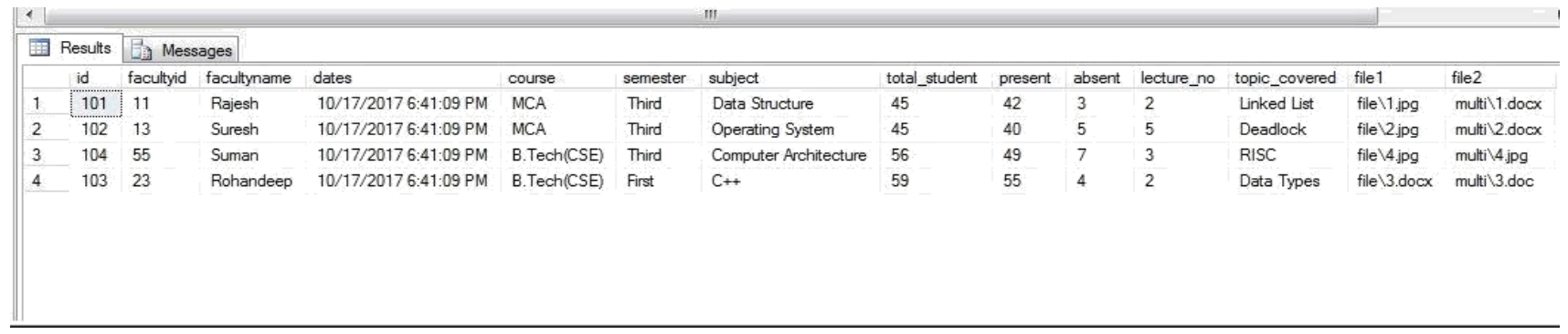

Figure 9 Data stored in table on SQL Server

As shown in figure 9 Data Synchronized from client to server. Data from access database automatically copied into sql server from client to server in order. Firstly the entries copied from client to server then new entry saved on server.

\subsection{Access Table Empty (All the Entries Shifted From Client(Access Table) To Server (Sql Server))}

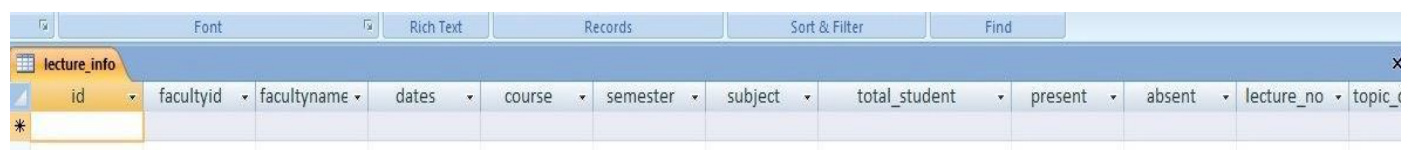

Figure 10. After data sink empty table of access database

Figure 10 shows the access empty table after data sink. 


\subsection{File Synchronization with database (Uploaded from client server)}

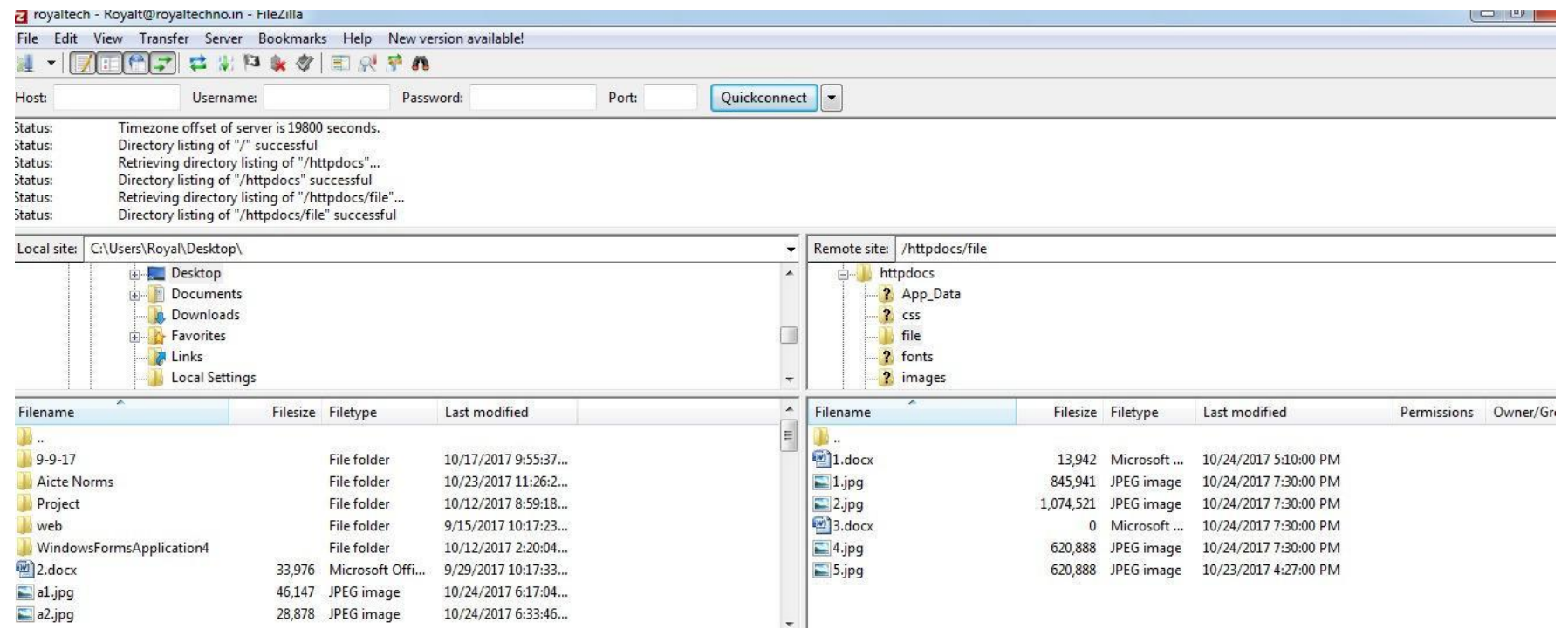

Figure 11. FTP showing files uploaded from client to server

Filezila FTP Print Screen showing file synchronized with database moved from client to server automatically (As shown in figure 11).

\section{CONCLUSION}

As the results are presented above with the help of print screen all the clients reliant on a single server. If that database becomes unavailable due to planned server downtime or from server failures, all of the remote workers will be disconnected from their data. Data is stored on to their system (user system). When the user connected to the internet data automatically sink from their client system to the server in serial order. This algorithm successfully works on data synchronization with image uploading.

\section{REFERENCE}

[1] www.cs.umb.edu/cs630/hd1.pdf

[2] en.wikipedia.org/wiki/Synchronization_(computer_science)

[3] https://coronet.iicm.tugraz.at/Dbase1/scripts/rdbh12.html

[4] Ari Trachtenberg, David Starobinski, and Sachin Agarwal," Fast PDA Synchronization Using Characteristic Polynomial Interpolation" INFOCOM 2002.

[5] B. Stasicki, K. Ehrenfried, L. Dieterle; K. Ludwikowski, M. Raffel," Advanced synchronization techniques for complex flow field investigations by means of PIV" 4th International Symposium on Particle Image Velocimetry Göttingen, Gernumerous, September 17-19, 2001.

[6] Saurav kumar Jha and Shoney Sebastian(2017), "An Algorithm to synchronize the local database with cloud Database", International Journal of Innovative Research in Advanced Engineering (IJIRAE), Issue 04, Volume 4.

[7] Matthias Wiesmann, Fernando Pedone, Andre Schiper, Bettina Kemme and Gustavo Alonso,"Database Replication Techniques: a Three Parameter Classification "Proceedings of 19th IEEE Symposium on Reliable Distributed Systems (SRDS2000), pages 206-215, Nürnberg, Gernumerous, October 2000. IEEE Computer Society. 